\title{
Qualidade da avaliação da acuidade visual realizada pelos professores do progirama "Olho no olho" da cidade de Marília, SP
}

\author{
Assessment of visual acuity evaluation performed by teachers \\ of the "Eye in eye" program in Marília-SP, Brazil
}

\author{
Lígia Issa De Fendi ${ }^{1}$ \\ Gustavo Viani Arruda ${ }^{2}$ \\ Ellen Carrara Fonseca ${ }^{3}$ \\ EvandroPortallupe Bosso ${ }^{4}$ \\ José Augusto Alves Ottaiano ${ }^{5}$
}

Trabalho realizado no Departamento de Oftalmologia da Faculdade de Medicina de Marília - FAMEMA - Marília (SP) - Brasil

Residente do Departamento de Oftalmologia da Faculdade de Medicina de Marília - FAMEMA - Marília (SP) - Brasil.

${ }^{2}$ Médico do Departamento de Radio-Oncologia da FAMEMA - Marília (SP) - Brasil

${ }^{3}$ Residente do Departamento de Oftalmologia da FAMEMA - Marília (SP) - Brasil.

${ }^{4}$ Médico titular do Departamento de Oftalmologia da FAMEMA - Marília (SP) - Brasil.

Doutor, Professor do Departamento de Oftalmologia da FAMEMA - Marília (SP) - Brasil.

Endereço para correspondência: Lígia Issa De Fendi. Rua Coronel José Brás, 205 - Apto. 72 - Marília (SP) CEP 17501-570

E-mail: lidefendi@hotmail.com

Recebido para publicação em 18.03.2007

Última versão recebida em 08.02.2008

Aprovação em 23.04.2008

Nota Editorial: Depois de concluída a análise do artigo sob sigilo editorial e com a anuência do Dr. Marinho Jorge Scarpi sobre a divulgação de seu nome como revisor, agradecemos sua participação neste processo.

\section{RESUMO}

Objetivos: Avaliar a qualidade e o ponto de corte $(\mathrm{AV} \leq 0,7)$ da acuidade visual verificada pelos professores, nos escolares da rede municipal de Marília participantes do programa "Olho no olho". Métodos: Estudo transversal em 604 escolares (1.208 olhos), pertencentes às escolas municipais de Marília, SP, realizado através do exame feito pelos professores treinados para o programa comparando-o com o exame realizado pelos oftalmologistas, assumindo estes como o padrão-ouro para comparação. A análise foi feita por olhos examinados. Calculamos a sensibilidade $(\mathrm{S})$, especificidade (E), valor preditivo positivo (VPP), valor preditivo negativo (VPN) e razão de verossimilhança (RV). Comparamos as médias das AV encontradas pelos professores com as dos oftalmologistas e uma curva ROC (Receiver Operating Characteristic Curve) foi gerada para avaliar se $\mathrm{AV} \leq 0,7$ foi o melhor valor para encaminhamento. Resultados: A média da AV verificada pelos professores foi de $0,70+/-0,16$ e a dos oftalmologistas foi de $0,88+/-0,2$ com uma diferença entre as médias de $0,18(\mathrm{p}<0,0001)$. A S, E, VPP, VPN e RVP foram de: $82 \%, 40 \%, 27 \%, 89 \%$ e 1,37, respectivamente. As taxas de falso positivo e negativo foram de 59,5\% e $18 \%$, respectivamente. Na curva ROC AV $\leq 0,7$ foi o melhor ponto para encaminhamento. Conclusões: Evidenciamos a importância da participação dos professores na melhoria da saúde ocular escolar. O teste realizado pelos professores teve satisfatória $\mathrm{S}$, com baixa $\mathrm{E}, \mathrm{VPN}$ e valores elevados de falsos positivos. Nossos dados confirmam que o melhor valor de corte para encaminhamento foi o valor de $\mathrm{AV} \leq 0,7$.

Descritores: Acuidade visual; Qualidade; Saúde escolar; Sensibilidade e especificidade; Criança

\section{INTRODUÇÃO}

Dados do Ministério da Educação ${ }^{(1)}$ indicam que o número de alunos na primeira série do ensino público fundamental é de quase 6 milhões. Entretanto, somente parte inexpressiva dessa população é submetida a algum tipo de avaliação oftalmológica antes de ingressar na escola. Números publicados pelo Conselho Brasileiro de Oftalmologia (CBO) mostram que no Brasil aproximadamente $20 \%$ dos escolares apresentam alguma alteração oftalmológica. Segundo o $\mathrm{CBO}, 10 \%$ dos alunos primários necessitam de correção por serem portadores de erros de refração: hipermetropia, miopia e astigmatismo; destes, aproximadamente 5\% têm 
redução grave de acuidade visual ${ }^{(1)}$. A prevenção e a detecção precoce de deficiências oculares são os melhores recursos para combate à visão subnormal e devem ser feitas, preferencialmente, na infância. Sendo a escola uma instituição com grande concentração de crianças, cabem aos profissionais da área da saúde escolar e ocular as ações de detecção e tratamento de baixa visão ${ }^{(2)}$. Para atingir o objetivo comum da saúde da criança em idade escolar é necessária a ação integrada larescola-comunidade ${ }^{(3)}$. Nos programas de triagem visual é importante estipular o critério de encaminhamento dos indivíduos como, por exemplo, o limite de visão a ser considerado. Esta preocupação resulta do fato de que este não pode ser tão alto para que não haja um número excessivo de crianças encaminhadas, gerando exames desnecessários, o contrário também é indesejável, pois pode deixar de lado crianças que tenham problemas oculares.

O objetivo deste estudo foi testar a qualidade da triagem realizada pelos professores do ensino fundamental da cidade de Marília. Para isto avaliamos a sensibilidade (S), especificidade (E), valor preditivo positivo (VPP), valor preditivo negativo (VPN) e razão de verossimilhança positiva (RVP) da acuidade visual medida pelos professores em escolares no ano de 2005 e 2006 tomando como base de comparação (padrãoouro) as medidas feitas pelos médicos do departamento de oftalmologia da Faculdade de Medicina de Marília (FAMEMA); Além disso, nosso estudo procurou avaliar se a acuidade visual $\leq 0,7$ é o melhor valor encontrado para o encaminhamento dos escolares ao nosso serviço de oftalmologia.

\section{MÉTODOS}

Estudo transversal realizado para avaliar a medida da AV realizada pelos professores nos escolares de 5 a 7 anos do ensino fundamental das redes pública da cidade de MaríliaSP, no ano de 2005-2006 e compará-lo com os resultados das medidas feitas pelos médicos oftalmologistas do departamento da FAMEMA. O objetivo desta comparação foi avaliar a sensibilidade, especificidade, valor preditivo positivo, valor preditivo negativo e razão de verossimilhança dos testes realizados pelos professores. Com objetivo de avaliar tanto os valores subestimados como os valores superestimados pelos professores, fizemos a análise por olhos examinados, dessa forma pudemos avaliar, de forma mais acurada e precisa, os exames feitos pelos professores na rede escolar. Neste período foram encaminhadas ao ambulatório de oftalmologia da FAMEMA 1.602 crianças, conforme os seguintes critérios:

- Escolares com AV igual ou menor do que 0,7 em pelo menos um dos olhos, com ou sem sinais e sintomas;

- Escolares com diferença de visão entre os olhos, de duas ou mais linhas, na escala optométrica de Snellen (ex. $\mathrm{OD}=0,5 \mathrm{e}$ $\mathrm{OE}=0,3$ ou $\mathrm{OD}=0,9$ e $\mathrm{OE}=0,7$ );

- Escolares portadores de estrabismo;

- Escolares que, apesar de visão normal, apresentaram, na observação do professor, sintomas de fadiga visual aos esfor- ços, dor de cabeça na região dos supercílios, lacrimejamento, tonturas e problemas acentuados de leitura e de escrita.

Considerou-se para análise uma amostra válida de 604 alunos com um total de 1.208 olhos examinados. Verificou-se que 306 alunos pertenciam ao sexo masculino com uma média de idade de 7,1 anos. Este estudo foi realizado durante a etapa de triagem do Programa de Avaliação Oftalmológica em Escolares, a cargo do Setor de Saúde Escolar da Secretaria Municipal de Saúde de Marília - SP, no período compreendido entre 2005-2006. O programa faz parte de uma campanha nacional idealizada pelo Conselho Brasileiro de Oftalmologia, com o apoio do Ministério da Saúde do Brasil. O projeto visa prestar assistência oftalmológica aos escolares do ensino fundamental da rede pública do município de Marília. Para as que se encaixam nos critérios acima citados, o programa possibilita o encaminhamento a serviços médicos especializados para exame oftalmológico detalhado. Na escola, professores treinados medem a AV através da tabela de optotipos "E" de Snellen a uma distância de seis metros, examinando cada olho separadamente, devendo a criança indicar as posições da letra "E". As crianças que usavam óculos foram examinadas sem e com óculos. O exame oftalmológico consistiu em uma consulta oftalmológica padrão, que é realizada rotineiramente pelos médicos oftalmologistas. Este exame incluiu: exame externo, motilidade ocular, reflexo pupilar, acuidade visual sem e com correção, refração sob cicloplegia, fundoscopia direta e biomicroscopia (quando necessário). A acuidade visual foi medida utilizando-se escala optotipos de Snellen a uma distância de 6 metros. A cicloplegia foi obtida com colírio de tropicamida a $1 \%$ e ciclopentolato $1 \%$, uma gota em cada olho de $10 \mathrm{em} 10$ minutos por duas vezes e o exame foi realizado 20 minutos após a última instilação. Caso a criança não fosse examinada em trinta minutos após a instilação do colírio, pingava-se uma nova gota, para manutenção do efeito cicloplégico ${ }^{(4)}$.

\section{Análise estatística}

Para os cálculos da S, E, VPP, VPN e RVP as medidas encontradas nos escolares pelos professores, com acuidade visual $\leq 0,7$ foram considerados como testes positivos e aqueles com $\mathrm{AV} \leq 0,7$ medida pelos oftalmologistas foram consideradas verdadeiros positivos, ou seja, padrão-ouro (Tabela 1). As médias encontradas nos exames foram calculadas para avaliar se houve diferença entre as médias dos professores e dos oftalmologistas, o teste $t$ Student foi usado para amostras pareadas, com $\mathrm{p}<0,05$ considerado significativo. Para tentar

$\begin{aligned} & \text { Tabela 1. Valores das medidas das AV encontradas em 1.208 olhos } \\
& \text { dos escolares avaliados pelos professores e os oftalmologistas }\end{aligned}$
\begin{tabular}{lccr} 
& Teste oftalmologista & Total \\
Teste professores & $\mathbf{A V}>\mathbf{0 , 7}(-)$ & $\mathbf{A V} \leq \mathbf{0 , 7}(+)$ & \\
$\mathrm{AV}>0,7(-)^{*}$ & 385 & 46 & 431 \\
$\mathrm{AV} \leq 0,7(+){ }^{* *}$ & 567 & 210 & 777 \\
Total & 952 & 256 & 1208 \\
${ }^{*}=\mathrm{AV}>0,7(-)=$ teste negativo; ${ }^{* *}=\mathrm{AV} \leq 0,7(+)=$ teste positivo \\
\hline
\end{tabular}


encontrar o valor de AV mais sensível e com menos falso positivo das medidas realizadas pelos professores foi construída uma curva ROC e calculada a área sob a curva para estimar sua acurácia com a atual medida $(\mathrm{AV} \leq 0,7)$.

\section{RESULTADOS}

A média da AV encontrada nos 1.208 olhos avaliados pelos professores foi de $0,70 \pm 0,16$, enquanto a média para $\mathrm{AV}$ medida pelos oftalmologistas foi de $0,88 \pm 0,20$. Houve diferença nas médias encontradas entre professores e oftalmologistas de 0,18 sendo altamente significativa $(p<0,0001)$, como mostrado na tabela 2 . Nos escolares com AV $\leq 0,7$ a média

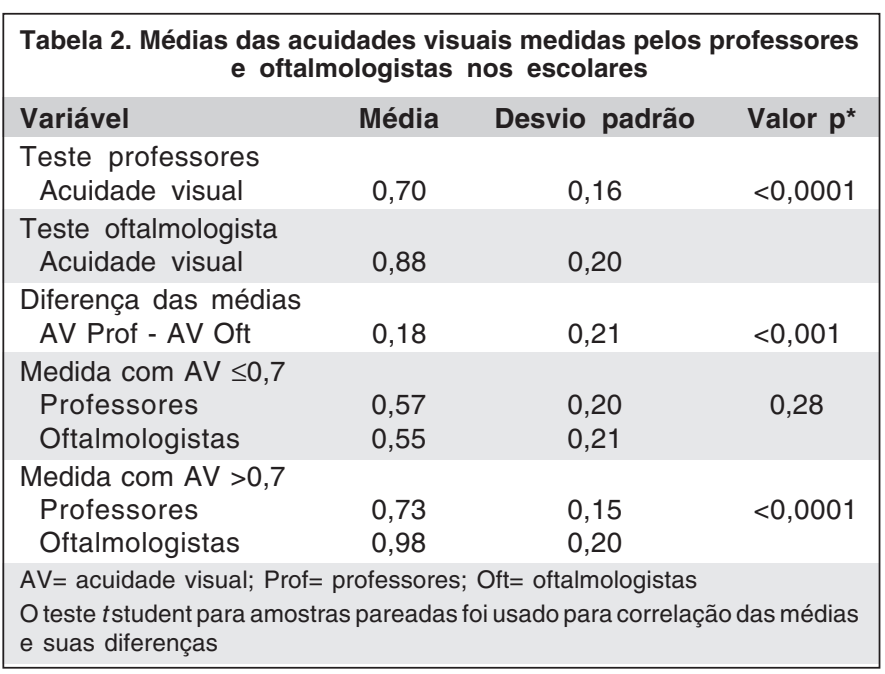

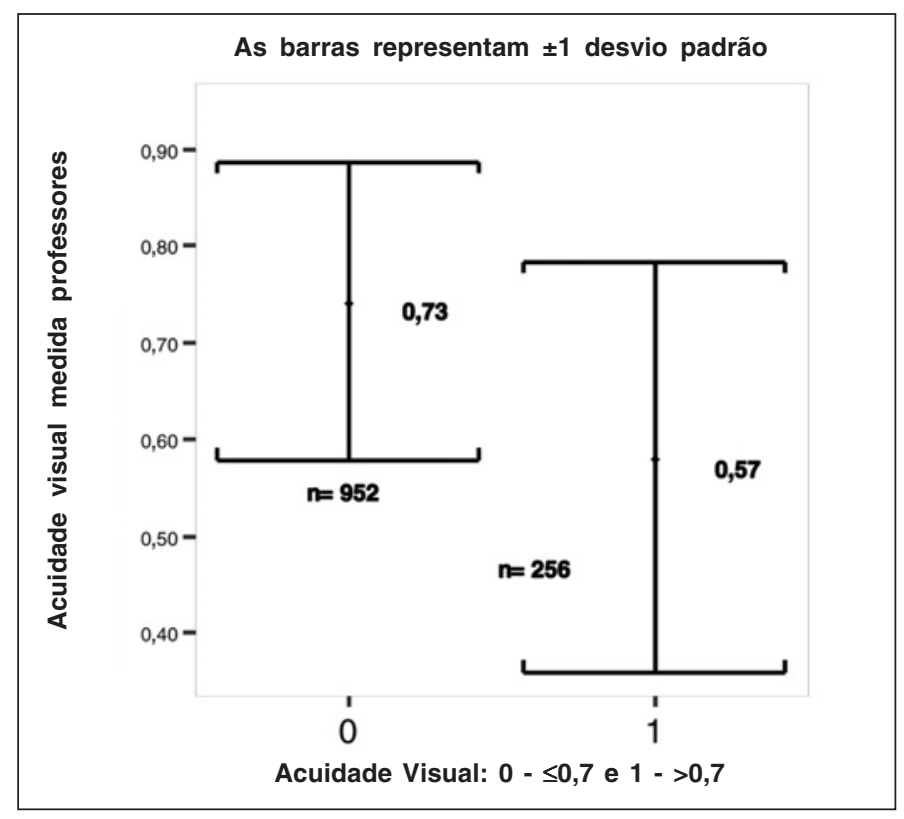

Figura 1 - Média e desvio padrão das medidas realizadas pelos professores nos 604 alunos (1.208 olhos) considerados com $A V \leq 0,7$ e $A V>0,7$ verificada pelos professores foi $0,57 \pm 0,20$ e pelos médicos $0,55 \pm 0,20$; naqueles com $\mathrm{AV}>0,7$ a média dos professores foi $0,73 \pm 0,15$ e dos oftalmologistas $0,98 \pm 0,02$. Dos 431 olhos avaliados como $\mathrm{AV}>0,7$ pelos professores, 385 foram confirmados pelo exame do oftalmologista. Não houve diferença estatística para as médias encontradas nos escolares com AV $\leq 0,7(p=0,287)$ entre professores $(0,57 \pm 0,20)$ e oftalmologis$\operatorname{tas}(0,55 \pm 0,20)$. Nos escolares com $A V>0,7$ houve significativas diferenças $(\mathrm{p}<0,0001)$ entre as acuidades visuais medidas pelos professores $(0,73)$ e oftalmologistas $(0,98)$ (Tabela 2$)$. A prevalência de acuidade visual diminuída, avaliada pelos oftalmologistas foi de 21,1\%. A S, E, VPP, VPN e RVP para encontrar acuidade visual diminuída nos escolares quando examinadas pelos professores foi de: $82 \%, 40 \%, 27 \%, 89 \%$ e 1,37 , respectivamente (Tabela 3 ). A taxa de falsos positivos e negativos nos exames dos professores foi de 59,5\% e $18 \%$ respectivamente. A razão de chance pré e pós-teste foram de

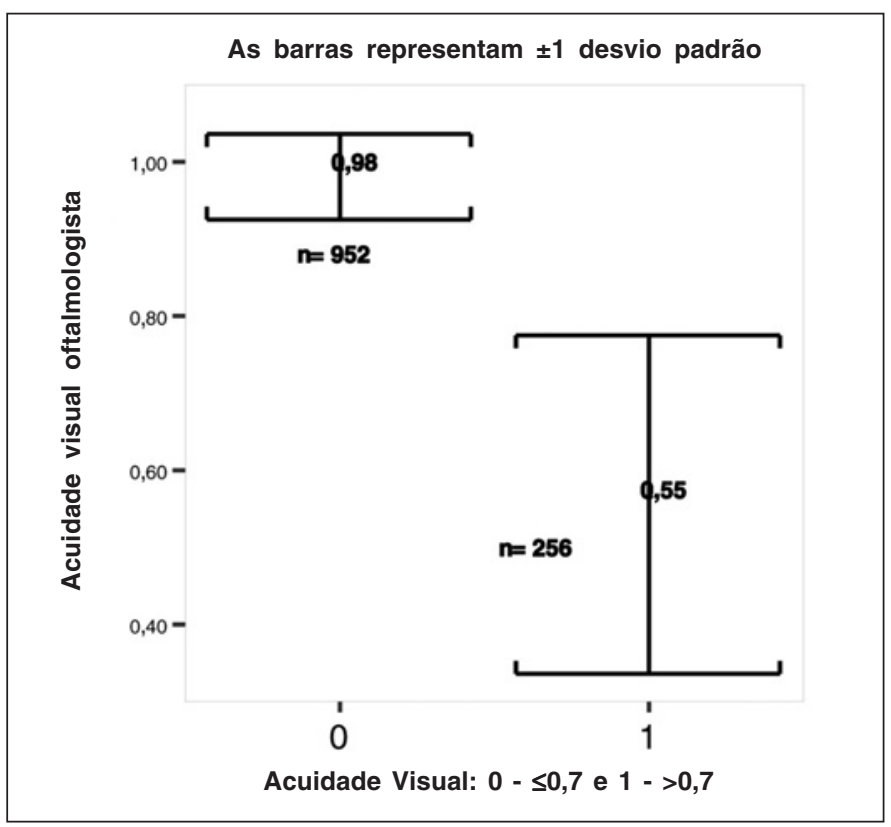

Figura 2 - Média da AV medida pelos oftalmologistas em 604 alunos (1.208 olhos) separados por AV $\leq 0,7$ e AV $>0,7$

\begin{tabular}{lc}
$\begin{array}{l}\text { Tabela 3. Valores do teste dos professores para acuidade visual } \\
\leq \mathbf{0 , 7} \text {, usando o teste dos oftalmologistas como padrão-ouro para } \\
\text { acuidade visual diminuída }\end{array}$ \\
$\begin{array}{l}\text { Resultados } \\
\text { Sensibilidade }\end{array}$ \\
$\begin{array}{l}\text { Especificidade } \\
\text { Valor preditivo negativo }\end{array}$ \\
Valor preditivo positivo & 82,00 \\
Razão de verossimilhança positiva & 40,40 \\
Falso positivo & 27,00 \\
Falso negativo & 89,00 \\
Razão de chance pré-teste & 1,37 \\
Razão de chance pós-teste & 59,00 \\
& 18,00 \\
\hline
\end{tabular}




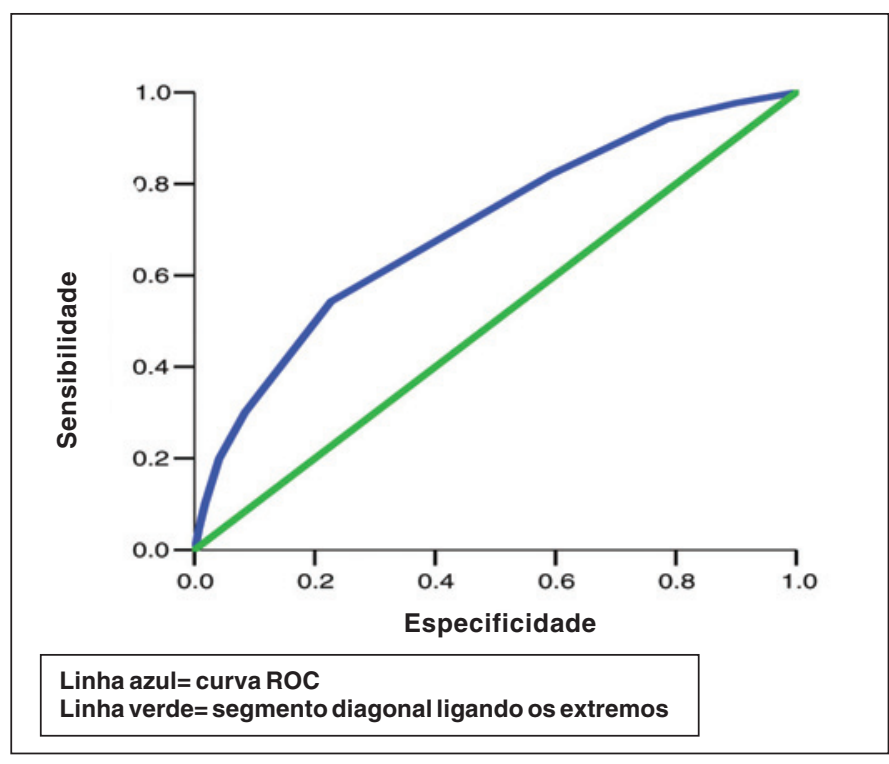

Figura 3 - Curva ROC evidenciando a sensibilidade e falsos positivos para os diferentes pontos de corte para AV reduzida

\begin{tabular}{|c|c|c|}
\hline \multicolumn{3}{|c|}{$\begin{array}{l}\text { Tabela da curva ROC. Representativa dos valores decimais comc } \\
\text { possíveis pontos de corte "cut-off" para acuidade visual reduzida } \\
\text { com suas sensibilidades e falsos positivos }\end{array}$} \\
\hline $\begin{array}{l}\text { Positivo se } \\
\text { menor ou igual }\end{array}$ & $\begin{array}{c}\text { Sensibilidade } \\
(\%)\end{array}$ & $\begin{array}{c}\text { Especificidade } \\
(\%)\end{array}$ \\
\hline 1,0 & 100 & 100 \\
\hline 0,9 & 97,7 & 90 \\
\hline 0,8 & 94 & 80 \\
\hline 0,7 & 82 & 59 \\
\hline 0,6 & 54,3 & 27,4 \\
\hline 0,5 & 30 & 8,4 \\
\hline 0,4 & 20 & 4,1 \\
\hline 0,3 & 10,2 & 1,8 \\
\hline 0,2 & 4,7 & 0,7 \\
\hline 0,1 & 1,6 & 0,3 \\
\hline 0 & 0 & 0 \\
\hline
\end{tabular}

$16 \%$ e $36 \%$. Através da curva ROC encontramos que a AV $\leq 0,7$ teve uma sensibilidade de $82 \%$ e uma taxa de falso positivo de $59 \%$, como observado na figura 3 .

Para os valores de AV a seguir a sensibilidade e a taxa de falsos positivos foram de: $\mathrm{AV} \leq 1,0(100 \%$ e $100 \%), \mathrm{AV} \leq 0,9(97,7 \%$ e $90 \%), \mathrm{AV} \leq 0,8$ ( $94 \%$ e $80 \%), \mathrm{AV} \leq 0,7$ ( $82 \%$ e $59 \%), \mathrm{AV} \leq 0,6$ (54,3\% e $27,4 \%), \mathrm{AV} \leq 0,5$ (30\% e $8,4 \%), \mathrm{AV} \leq 0,4$ ( $20 \%$ e $4,1 \%)$, $\mathrm{AV} \leq 0,3(10,2 \%$ e $1,8 \%), \mathrm{AV} \leq 0,2(4,7 \%$ e $0,7 \%), \mathrm{AV} \leq 0,1(1,6 \%$ e $0,3 \%$ ), como mostrado na figura 3 e tabela da curva ROC.

\section{DISCUSS ÃO}

Em países em desenvolvimento, as condições socioeconômicas e culturais dificultam o acesso da criança ao exame oftalmológico antes de seu ingresso na escola. Para preencher esta lacuna, são realizadas campanhas de avaliação da acuidade visual dos escolares, já no primeiro ano escolar, procurando detectar possíveis alterações oculares a fim de que elas sejam corrigidas ou para prevenir piora ${ }^{(3-5)}$. A promoção da saúde ocular apresenta o princípio básico de melhoria da qualidade de vida do indivíduo, mediante a obtenção da capacidade visual que lhe permita o desenvolvimento de suas potencialidades e participação na sociedade ${ }^{(6)}$. A prevalência de AV reduzida em nosso estudo foi de $21 \%$, valor este um pouco acima dos resultados apresentados por outros autores ${ }^{(2,5,7-9)}$. Este dado provavelmente deve ter ocorrido devido a nossa análise ter sido feita por olhos examinados. A prevalência de $A V$ reduzida $(\mathrm{AV} \leq 0,7)$ e alterações oculares encontrada em estudo com pré-escolares e escolares da $1^{\underline{\underline{a}}}$ a $8^{\underline{\underline{a}}}$ séries de escolas municipais, estaduais e particulares da cidade de Ibiporã - PR, foi de $14,59 \%^{(10)}$. Na Colômbia, a prevalência foi de $19,6 \%$ de alunos com dificuldade visual (os autores não especificam o limite de $\mathrm{AV})^{(7)}$. Na Argentina foi encontrado uma prevalência de $18,69 \%$ de AV reduzida (AV $\leq 0,7)$ entre escolares do primeiro grau; entre os estudantes da primeira série a prevalência de AV reduzida foi de $18,09 \%{ }^{(8)}$. Como nossa análise objetivou avaliar a qualidade do teste para AV reduzida realizada pelos professores em escolares, decidimos fazer a análise por olhos examinados, pois se fizéssemos a análise por paciente não conseguiríamos avaliar as taxas de falso negativo, especificidade e valor preditivo negativo. Dentre os olhos examinados a AV reduzida foi confirmada em 210 . Ou seja, a taxa de verdadeiro-positivo de $27 \%$. Conseqüentemente, o falso positivo foi de $59,5 \%$, o que implicou num grande número de encaminhamentos desnecessários. Os índices de falsos positivos encontrados no presente estudo foram um pouco superiores aos apresentados por um estudo realizado em Ibiporã - PR e em Limeira - SP, onde os índices foram de $54,5 \%$ e de $49,3 \%$, respectivamente ${ }^{(9-10)}$. Este fator pode ter ocorrido devido à curva de aprendizagem do teste de AV, a criança chegava ao consultório oftalmológico conhecendo o teste de $\mathrm{AV}$ e, portanto, com uma possibilidade maior de fornecer a informação correta. Outra hipótese a ser levada em consideração é que os professores subestimaram a AV. Em nosso estudo a média encontrada para a AV em 1.208 olhos medida pelos médicos foi de 0,88 sendo 0,18 maior $(p<0,001)$ do que a encontrada pelos professores. Além disso encontramos para os alunos com AV $\leq 0,7$ (256 olhos) uma média de $0,57 \pm 0,20$ na avaliação dos professores, na avaliação dos oftalmologistas a média foi de $0,55 \pm 0,20 \quad(\mathrm{p}=0,28)$. Nos escolares com AV $>0,7 \quad(\mathrm{n}=952$ olhos) a média foi de $0,73 \pm 0,15$ na avaliação dos professores, enquanto na avaliação dos oftalmologistas a média foi de $0,98 \pm 0,20(p<0,0001)$, confirmando nossa hipótese. A provável explicação para esse dado é que existe uma tendência dos professores de encaminhar os casos duvidosos ${ }^{(9,11)}$, desta forma ocorre uma redução dos valores da AV nos escolares que realmente não apresentem AV reduzida. No entanto, mesmo subestimando a $\mathrm{AV}$ o valor preditivo negativo foi de $89,3 \%$. A razão de chance pré-teste e pós-teste foram de $26 \%$ 
e $36 \%$, evidenciando que o exame foi útil como exame de triagem levando-se em conta a prevalência da AV reduzida nesta população e os possíveis bias envolvidos com o teste. Encontramos uma razão de verossimilhança positiva maior do que $1(1,37)$, mostrando que o exame feito pelos professores foi útil em detectar crianças com AV reduzida, este valor é considerado satisfatório, mas poderia ser mais alto. $\mathrm{O}$ valor da RVP pode ser atribuído às medidas de $\mathrm{AV}$ subestimadas pelos professores, levando a um maior número de escolares a serem examinados pelos oftalmologistas para se obter um exame positivo. Atualmente os programas que fazem parte da campanha nacional idealizada pelo CBO com apoio do Ministério da Saúde do Brasil utilizam, como ponto de corte para encaminhamento a serviços médicos especializados o valor de $\mathrm{AV} \leq 0,7$. Em nossa análise, confirmamos ser o melhor valor para encaminhamento dos escolares a $\mathrm{AV} \leq 0,7$, analisando a curva ROC, valores acima deste como, por exemplo, $\mathrm{AV} \leq 0,8$ produziria uma sensibilidade de $94 \%$ com uma taxa de falso positivo de $80 \%$ tornando inviável o teste como exame de triagem para detecção precoce. Por outro lado, valores abaixo de 0,7 diminuiriam a sensibilidade a níveis muito reduzidos inviabilizando o teste com o objetivo de triagem, já que somente os escolares com níveis acentuados de AV reduzida seriam encaminhados perdendo-se o objetivo de detectar os casos iniciais potencialmente passíveis de correção ou prevenção de piora pelo oftalmologista.

\section{CONCLUSÃO}

O presente estudo evidencia a importância da participação dos professores na melhoria da saúde ocular escolar. Nossos dados mostram que os exames de detecção precoce de AV reduzida realizados pelos professores nos escolares geralmente subestimam os valores realmente apresentados por eles quando comparados aos valores obtidos pela avaliação dos oftalmologistas. Conseqüentemente produzindo um teste de detecção precoce de AV reduzida com uma satisfatória sensibilidade, às custas de uma baixa especificidade, valor preditivo negativo e valores elevados de falsos positivos. Nossos dados confirmam que o melhor valor de corte para encaminhamento sob suspeita de AV reduzida em escolares foi o valor de AV $\leq 0,7$. Deste modo, ressaltamos a importância do papel do professor na identificação do aluno com problemas de visão, fazendo-se necessária a orientação dos docentes, visando ampliar seu conhecimento e compreensão a respeito do papel fundamental que desempenham nas ações de saúde ocular na escola.

\section{ABSTRACT}

Purpose: To assess quality and cut-off point $(\mathrm{VA} \leq 0.7)$ of the examinations performed by teachers to detect reduced visual acuity (VA) in schoolchildren participants of the project called "Eye in eye " in Marilia-SP, Brazil. Methods: Visual acuity measurements were performed by trained teachers using Snellen's chart. The children with VA $\leq 0.7$ in one of the eyes were referred to an ophthalmic examination. The ophthalmic examination was considered gold standard to assess quality and cut-off point of the examinations performed by teachers. VA test was performed in 604 schoolchildren of state public schools (1,208 eyes). Analyses were based on examined eyes. We calculated sensitivity (S), specificity (E), positive predictive value (PPV), negative predictive value (NPV) and likelihood rate (LR). We compared the means obtained by the teachers' examinations to means of the ophthalmologist's examinations. ROC curve was produced to evaluate whether VA lower than 0.7 is the best value for referral to an ophthalmic examination. Results: VA means obtained by teachers and ophthalmologic examinations were $0.70 \pm 0.16$ and 0.88 \pm 0.2 respectively. The difference between teachers' and ophthalmologists' examinations was $0.18(\mathrm{p}<0.0001)$. S, E, PPV, NPV e LR were: $82 \%, 40 \%, 27 \%, 89 \%$ and 1.37 , respectively. False positive and negative rates were $59.5 \%$ and $18 \%$. ROC curve evidenced that visual acuity of 0.7 was the best cut-off point to refer schoolchildren to an ophthalmic exam. Conclusions: We demonstrated the importance of the teachers' participation in improved schoolchildren ocular health. The examination performed by teachers obtained a satisfactory $\mathrm{S}$ with low E, NPV and high values of false positive results. The best cut-off point to refer schoolchildren to an ophthalmic examination was VA of 0.7 .

Keywords: Visual acuity; School health; Sensitivity and specificity; Child

\section{REFERÊNCIAS}

1. Alves MR, Kara-José N. Manual de orientação. Campanha Veja Bem Brasil, 1998. São Paulo: Conselho Brasileiro de Oftalmologia; 1998.

2. Fechine ADL, Cardoso MVL, Pagliuca LMF. Prevenção e detecção de distúrbios oftalmológicos em escolares. Ped Atual. 2000;13(4):21-5.

3. Kara-José N, Alves MR. Problemas oculares mais freqüentes em escolares. In: Conceição JAN, coordenador. Saúde escolar: a criança, a vida e a escola. São Paulo: Sarvier; 1994. p.195-203.

4. Alves MR, Kara-José N. O olho e a visão: o que fazer pela saúde ocular das nossas crianças. Petrópolis: Vozes; 1996. p.151.

5. Temporini ER. Ação preventiva em problemas visuais de escolares. Rev Saúde Pública. 1984;18(3):259-62.

6. Temporini ER. Promoção da saúde ocular. Arq Bras Oftalmol. 1999;62(1):82-4.

7. Guerrero V R, Martinez C CE, Wooley L. Defectos de refracción y rendimiento academico en la escuela primaria. Colomb Med. 1989;20(1):8-10.

8. Pastorino N, Penerini Y. Programa de detección de déficit de la agudeza visual en escolares sin patologia ocular aparente. Arch Argent Pediatr. 1998;96(4):236-41.

9. Castro RS. Triagem visual e assistência oftalmológica em pré-escolares da cidade de Limeira (SP), 1995. Campinas (SP): UNICAMP; 1997.

10. Schimiti RB, Costa VP, Gregui MJF, Kara-José N, Temporini ER. Prevalence of refractive errors and ocular disorders in preschool and schoolchildren of Ibiporã - PR, Brazil (1989 to 1996). Arq Bras Oftalmol. 2001;64(5):379-84.

11. Kara-José N, Temporini ER. Avaliação dos critérios de triagem visual de escolares de primeira série do primeiro grau. Rev Saúde Pública. 1980;14:205-14. 